\title{
Mais quel est donc le rôle physiologique de la guanylyl cyclase-C intestinale?
}

Mais à quoi peut donc servir la guanylyl cyclase-C (GC-C) intestinale, hormis son rôle de récepteur de certaines entérotoxines (désignées heatstable enterotoxin ou STa) de E. coli relayant ainsi leur action diarrhéique parfois fatale? Compte tenu de la forte mortalité liée à ce phénomène (plus de 800000 morts par an, à l'échelle mondiale), cette protéine transmembranaire, seul récepteur identifié des STa, suscite un intérêt certain. Le mécanisme d'action est aujourd'hui en partie élucidé : l'activation de GC-C par STa augmente le GMPc dans l'épithélium intestinal, déclenchant ainsi une cascade d'événements dont la conséquence est l'accumulation d'eau et de sels dans la lumière gastro-intestinale. Ce processus mettrait en jeu une protéine kinase dépendante du GMPc, la phosphorylation puis l'ouverture des canaux chlorure CFTR (cystic fibrosis transmembrane conductance regulator). La question du rôle physiologique de la GC-C est d'autant plus actuelle que deux peptides intestinaux capables de se lier à la GC-C et structurellement proches de STa, la guanyline et l'uroguanyline, ont fait récemment leur apparition sur la scène. En outre, le contrôle du problème de santé publique lié à l'infection bactérienne par E. coli, nécessite d'élucider le rôle de GC-C dans des conditions physiologiques normales. Dans ce contexte, l'invalidation du gène codant pour le récepteur GC-C réalisée récemment pouvait constituer une approche particulièrement séduisante pour aborder ce problème [1]. Par ailleurs, les outils de la biologie moléculaire permettent progressivement à l'uroguanyline et à la guanyline de sortir de l'ombre du système digestif. Le clonage récent des gènes et l'étude de la localisation des peptides au niveau cellulaire, et tout le long du tractus digestif, contribuent aujourd'hui à une meilleure compréhension de leur rôle physiologique [2, 3].

L'objectif de mieux percevoir le rôle du récepteur GC-C par son invalidation génique chez la souris n'a pas été réellement atteint au vu du phénotype des souris $G C-C^{-2}$. Ces souris apparaissent a priori physiologiquement "normales " voire "supranormales", l'absence de GC-C intestinale les ayant rendues résistantes à l'action des entérotoxines STa. Ainsi, alors que les souris $G C_{-} C^{4 /+}$ et $G C_{-}-C^{4--}$ meurent quelques jours après avoir été infectées par une souche de E.coli productrice de STa, les souris $G C-C^{L-}$ sont indemnes pour la plupart et ne présentent aucun symptôme diarrhéique. Confirmant ce phénotype, une accumulation de fluide est observée dans la lumière intestinale des nouveau-nés normaux de trois jours ayant reçu par voie orale l'entérotoxine STa, les souris $G C-C^{-/}$ étant encore une nouvelle fois épargnées. Hyper-résistantes, elles le sont devenues, puisque même un important changement dans le régime alimentaire (variations du taux de protéines, de matières grasses, de $\mathrm{NaCl}$, l'ajout de sucrose, de $\mathrm{KCl}$ ) ne semble pas avoir d'effet fatal. Ainsi, la question posée reste entière : pourquoi un récepteur qui conduit à une mortalité importante chez des jeunes animaux a-t-il été conservé au cours de l'évolution ? Existerait-il une condition délétère non encore identifiée, commune à toutes les espèces, pour laquelle la présence d'un récepteur GC-C fonctionnel est indispensable à la survie, en particulier l'attaque par d'autres agents pathogènes plus sévères que STa?

Mais alors, qu'en est-il des ligands naturels du récepteur GC-C intesti- nal ? Découverte la première comme ligand naturel de GC-C, la guanyline, peptide de 15 acides aminés identifié dans l'intestin de rat (50\% d'identité avec le STa), s'est vite trouvée une associée en l'uroguanyline, isolée dans l'urine d'oppossum, et présentant plus de $60 \%$ d'identité avec la guanyline. Elles ont été récemment localisées dans les différentes parties et cellules de l'intestin. Qu'elle soit sous la forme de précureur de 106 acides aminés, ou sous la forme mûre de 15 acides aminés, la structure de l'uroguanyline de souris est très proche de celles de la protéine humaine et de rat [2]. Avec le rein, le tractus digestif est le tissu le plus riche en uroguanyline: les transcrits sont détectés en majorité dans l'épithélium de l'intestin grêle (duodénum, jéjunum et iléon), le plus souvent au niveau des villosités. A l'échelle cellulaire, la protéine semble exclusivement confinée dans les cellules endocrines de type entérochromaffine qui synthétisent aussi la sérotonine, la substance $\mathrm{P}$ et les enképhalines [3]. Observation qui a probablement son importance, une toute autre carte d'identité caractérise la guanyline. Absente du rein, elle est retrouvée en abondance dans le tractus digestif, mais c'est le jéjunum, l'iléon, le cæcum et surtout le côlon qui en sont les plus riches. Elle y est détectée aussi bien dans les cellules villositaires que cryptiques et, autre singularité, exclusivement dans les cellules sécrétrices de mucus [2]. Cette complémentarité dans la distribution cellulaire et tissulaire de l'uroguanyline et de la guanyline confirme l'hypothèse d'un rôle spécifique pour chacune d'elles : la guanyline était présumée régler plutôt les fonctions sécrétrices hydroélectrolytiques intestinales, alors que l'uro- 
guanyline présente dans le sang et l'urine était plutôt considérée comme un médiateur endocrine entre l'intestin et le rein. Aujourd'hui, la présence de l'uroguanyline dans des régions intestinales exprimant de grandes quantités de GC-C soulève la question de son rôle dans le contrôle de la sécrétion de chlorures et de bicarbonates par l'intestin grêle. En outre, l'uroguanyline pourrait être le facteur intestinal recherché pour son activité natriurétique en réponse à une charge orale de sel. Encore une énigme dont on peut espérer trouver la solution par des études d'invalidation génique!

B.A.
1. Schulz S, Lopez MJ, Kuhn M, Garbers DL. Disruption of the guanylyl cyclase-C gene leads to a paradoxal phenotype of viable but heatstable enterotoxin-resistant mice. J Clin Invest $1997 ; 100: 1590-5$.

2. Whitaker TL, Witte DP, Scott C, Cohen MB. Uroguanylin and guanylin: distinct but overlapping patterns of messenger RNA expression in mouse intestine. Gastroenterology 1997; 113: 1000-6. 3. Perkins A, Goy MF, Li Z. Uroguanylin is expressed by enterochromaffin cells in the rat gastrointestinal tract. Gastroenterology 1997; 113: 1007-14.

\section{BRÈVES}

Un scénario moléculaire pour expliquer l'effet toxique du glucose supraphysiologique sur la cellule $\beta$ pancréatique! Par quel mécanisme une concentration supraphysiologique chronique de glucose conduitelle à la répression du gène de l'insuline, comme dans le diabète de type II, par exemple? Aujourd'hui, en se fondant sur des études physicochimiques et fonctionnelles in vitro, une équipe du Massachusetts propose un scénario dans lequel le facteur de transcription $\mathrm{C} / \mathrm{EBP} \beta$ jouerait le premier rôle. Le facteur $\mathrm{C} / \mathrm{EBP} \beta$, qui appartient à une famille de protéines nucléaires caractérisées par un domaine de dimérisation (motif leucine zipper) et un domaine basique de liaison à l'ADN, est déjà impliqué dans la croissance et la différenciation cellulaires, et la spécificité tissulaire d'expression de gènes $\left(m / s n^{\circ} 5\right.$, vol. 6 , p. 486). Dans la cellule $\beta$ pancréa- tique, $\mathrm{C} / \mathrm{EBP} \beta$ interagirait directement avec la protéine $\mathrm{E} 47$, un facteur de transcription de la famille $\beta \mathrm{HLH}$ (basic helix-loop-helix) qui est un transactivateur du gène de l'insuline, et abolirait son potentiel transactivateur [1]. Observations importantes à l'origine de ce modèle, C/EBP $\beta$ est synthétisé par la cellule $\beta$ pancréatique, et une concentration supraphysiologique de glucose stimule la synthèse de $\mathrm{C} / \mathrm{EBP} \beta$ dans un modèle de cellules $\beta$ pancréatiques en culture, à court terme (72 heures) aussi bien qu'à long terme (plusieurs semaines). Cet effet s'accompagne d'une inhibition de la synthèse d'insuline. Le promoteur du gène de l'insuline (de rat et d'homme) contient une région très spécifique (désignée boîte EBP) capable de lier le facteur C/EBP qui confère l'activation du gène de l'insuline par $\mathrm{C} / \mathrm{EBP} \beta$ dans des cellules non $\beta$ pancréatiques (fibroblastiques ou rénales), mais il la réprime dans les cellules $\beta$ pancréatiques et une mutation invalidante de la boîte EBP n'abolit que partiellement cette répression génique. C'est en fait l'interaction directe de $\mathrm{C} / \mathrm{EBP} \beta$ (par sa séquence leucine zipper) avec E47 (contenant une répétition de leucines au niveau d'un domaine d'activation) qui serait à l'origine de l'action répressive de $\mathrm{C} / \mathrm{EBP} \beta$ sur le gène insuline. L'altération de la fonction endocrine de la cellule $\beta$ pancréatique liée à l'hyperglycémie chronique du diabète de type II pourrait donc être essentiellement le fait de la protéine C/EBP $\beta$. Cette protéine serait donc impliquée dans le développement et de la progression de la maladie?

[1. Lu M, et al.J Biol Chem 1997; 272 : 28349-59.] 